\title{
Early Order Completion Contract Approach to Minimize the Impact of Demand Uncertainty on Supply Chains
}

\author{
Hing Kai Chan, Senior Member, IEEE, and Felix T. S. Chan
}

\begin{abstract}
Many optimization techniques have been proposed over the years to improve the performance of supply chains. Although these approaches have been shown to be effective, most of them were developed without considering uncertainties in supply chains to simplify the analysis. In fact, uncertainties can deteriorate the performance of supply chains, such as increase in total cost, or drop in fill rate, of the whole system. In distributed supply chains, participating members are not under a sole control by a central authority, the problem is even more stringent due to incomplete information sharing, or so called asymmetrical information sharing. One way to improve the system performance is to achieve coordination among participating parties through establishment of contracts. The objectives of this paper are i) to evaluate the effects of demand uncertainty in a distributed supply chain, which is modelled as an agent-based system; ii) to propose a coordination mechanism with early order completion contract to minimize the negative impacts of demand uncertainty; and iii) to present associated simulation results. Performance of the system is measured in terms of costs and fill rate. Simulation results indicate that the proposed contract approach is able to improve the performance measures of the system.
\end{abstract}

Index Terms - Contract, coordination, simulation, supply chain management, uncertainty.

\section{INTRODUCTION}

$\mathbf{S}$ UPPLY chains consist of a "network of participating organizations through upstream and downstream linkages, in different processes and activities that produce value in the form of products and services in the hands of the ultimate consumer" [1]. The present era, which is witnessing the growth of globalization, has forced the increase in entities flow and overall complexity of supply chain. And hence the uncertainty in production management often seems to grow as well [2]. Uncertainty takes the form of unforeseen demand variability, which in turn creates problems in planning, scheduling, and control of downstream entities [3]. Although safety stock inventory can improve the reliability of delivery performance by providing a buffer for uncertain upstream supply chain [4], which only transfers an additional inventory holding cost towards upstream, therefore it may not be the most effective way to deal with uncertainties. In

Manuscript received July 14, 2005; revised November 3, 2005 and December 29, 2005

The authors are with the Department of Industrial and Manufacturing Systems Engineering, The University of Hong Kong, Pokfulam, Hong Kong (e-mail: hkchan@ieee.org).

Digital Object Identifier 10.1109/TII.2005.864217 other words, a dynamic inventory replenishment approach may be more appropriate if the environment is uncertain.

The main objectives of this paper are threefold. Firstly, to evaluate the impacts of demand uncertainty in an agent-based distributed supply chain. Demand uncertainty in this paper is obtained using a normal distribution function, which will be presented in Section III. Secondly, a coordination mechanism with an early order completion contract, which aims to minimize the negative impacts of the demand uncertainty, is proposed. "Early order completion" means a retailer can request a supplier to complete an inventory replenishment order with predefined partial quantity, rather than waiting for the "full" quantity of the order to be completed. This practice may be helpful to improve supply chain performance because back orders can be filled immediately. Nevertheless, impacts of early order completion have not been addressed so far. Finally, simulation results based on the proposed algorithm will be presented. Performance of the system is measured in terms of costs and retailer's fill rate (or sometimes known as demand fill rate). These two performance measures are in fact represented under the two performance metrics as defined by the Supply Chain Operations Reference (SCOR) model [5]. These are considered as important measures for supply chain performance and hence they are employed as performance indictors in this simulation study.

Although the early order completion concept is useful, an order cannot be executed earlier arbitrarily without satisfying the constraints of a supply chain. Otherwise, the whole system may become out of control or can be called as having chaotic behavior. To overcome this problem, contract between the supplier and retailer can be established in order to guide the actions of the members through a coordination mechanism. This practice provides flexibility to both supplier and retailer. With this underlying principle, present paper sets out to investigate an early order completion in the form of contract with a proposed coordination mechanism on supply chain performances, subject to demand uncertainty. The organization of this paper is as follows. Section II reviews key literature and Section III presents the supply chain model along with its performance measures. The proposed coordination mechanism with early order completion contract will be described in this section as well. Section IV summarises the simulation results in this study, including the effects of demand uncertainty. This set of study is also treated as control experiments for the simulation with early order completion contract. Section V is the concluding section along with proposed areas for future research. 


\section{LITERATURE REVIEW}

\section{A. Uncertainties in Supply Chains}

Treating uncertainty is an important issue in supply chain modelling, and analysis of the system's performance [6]. Greater supply uncertainty increases the need for vertical integration while greater demand uncertainty increases the reliance on outsourcing in a multistage supply chain [7]. Petrovic et al. [8] attempted to examine the effects of a serial supply chain in uncertain environment and identified the sources of uncertainty were customer demand and supplier reliability. The authors concluded that uncertain customer demand and uncertain supply delivery along the supply chain have great impact on supply chain behavior. Apart from demand uncertainty and supplier reliability as mentioned above, other kind of uncertainties also exist along supply chain. For example, Geneste $e t$ al. [9] suggested a method to consider the uncertainty of subcontracted orders through a customer-subcontractor negotiation process. However, most traditional examinations of uncertainty in operations management have been based on, or identified the root as, demand uncertainty [10]. Therefore, demand variability is also the source of uncertainty in this study.

The main objective of any inventory policy is to minimize the average total supply chain cost over an infinite time horizon [11]. Considering the cost, Zhao and Xie [12] evaluated the impact on forecasting model that was caused by uncertain demand, which was basically modelled as a normal distribution function, in a capacitated supply chain. Simulation results indicated that forecasting error influences supply chain performance significantly in terms of total cost, total suppliers cost, and total retailers' cost of the supply chain. Zhao et al. [13] proposed that early order commitments by retailers would benefit most in terms of cost reduction, and service improvement of a similar supply chain which was reported in Zhao and Xie [14]. Although Zhao et al. [13] had concluded that early order commitments by retailers would be helpful to improve supply chain performances; in fact, it is not always easy to implement such concept under uncertain environment (will be explained later). On the other hand, coordination among various entities of supply chain may help to improve the performance of supply chain subject to uncertainties.

\section{B. Coordination in Supply Chains}

Coordination is a means to help supply chain members to counter react with uncertainties, and it is an important issue in a supply chain with multiple organizations [15]. In fact, supply chain management relates to the coordination of products and information flows among various entities in the system [13]. Lee et al. [16] discussed and claimed that information that is transferred in the form of "order" tends to be distorted in supply chains and can misguide upstream members in both their inventory and production decisions. The magnitude of distortion tends to increases as one move upstream along a supply chain, which can results in a phenomenon known as the "Bullwhip Effect". Consequently, additional inventory and production costs may be incurred. Coordination among supply chain members can be beneficial under such situation in reducing the inventory and hence cost.
Due to their complex interactions, supply chain members can increase their own profits by coordinating with each others in order to fulfill the system goals [17]. In fact, uncertainties lead to a greater loss to the members of a supply chain, which makes coordination as a tool under uncertain environments to increase supply chain's flexibility [3]. Facing uncertain demand, retailers prefer to place an order late in most case in order to gather enough time to collect more information to reduce demand forecast error [18]. However, this results in insufficient production times and hence production cost (including penalty cost) would probably be increased. Chen and Xu [18] advocated that this conflict exists universally in supply chains. That's why early order commitment as discussed before is not easy to be realized, unless a retailer is motivated to do so by additional benefit or incentive.

In fact, coordination can also exist in the form of contracting. For example, if a contract allows a retailer to instruct a supplier to deliver a quantity of product which is within a predefined range as established in the contract, then the retailer may be able to request less quantity before shipment because of unexpected low demand and, hence, inventory cost can be reduced. On the other hand, if the supplier's capacity is uncertain, the supplier may be able to complete the order with less quantity which still within the predefined range, and hence no penalty or lateness cost will be charged. Therefore, contract approach may beneficial to all related parties, if they are established well.

However, little literature exists regarding how specific parameters in a contract can provide [19]. In fact, such contract "provides flexibility with no explicit penalty for exercise, but uses constraints as a way to motivate appropriate behavior" [19]. Refer to the above example again, a set of solutions is defined once a contract is formed and no fixed solution is required. The actual solution is a result of an interactive and iterative procedure. Traditional mathematical approach, which attempts to find the optimal value, is therefore not an effective tool to adopt this philosophy for reacting with uncertainties. In fact, it is meaningless to find the optimal solution in many applications, such as the simulation study with uncertainty in this paper because uncertainty is an uncontrollable factor that is not easy to be represented by mathematical modelling, i.e., an optimal solution seems never exist.

\section{Agent-Based Studies in Supply Chains}

It is not surprising that distributed problem solving is a new paradigm in supply chains studies [20]. Among the available distributed problem solving techniques, agent-based coordination problem is the most popular one for solving supply chain problems [21]. One of the reasons is that in a multi-agent framework, agents are able to share information among them. In supply chain studies, Jung and Jo [22] proposed an agent-based coordination framework, which could satisfy various preferences of buyers and sellers. Kimbrough et al. [23] showed that agent-based supply chains could reproduce the beer games problem as reported by Sterman [24], which is essentially the Bullwhip Effect as coined by Lee et al. [16]. Kimbrough et $a l$. [23] concluded that intelligent agents are capable to track demand, eliminate the Bullwhip effect, discover the optimal policies (where they are known), and find good policies under 


\begin{tabular}{|c|c|c|c|}
\hline Description of events & Agent which announces the job & & Other agents \\
\hline [a] Order is needed, announce job to other agents & Job Announcement Agent & $\rightarrow$ & Job Reception Agent \\
\hline [b] Job Reception Agent relays the job to Bid & & & \\
\hline Evaluation Agent & & & \\
\hline [c] Other agents are considered to submit a bid & & & Bid Evaluation Agent \\
\hline [d] Other agents submit a bid according to their & \multirow[b]{2}{*}{ Bid Reception Agent } & & \multirow[b]{2}{*}{ Bid Evaluation Agent } \\
\hline own conditions & & & \\
\hline $\begin{array}{l}\text { [e] Deadline for bid submission is reached (no } \\
\text { more bids will be accepted) }\end{array}$ & Bid Reception Agent & & \\
\hline \multicolumn{4}{|l|}{ [f] Notify Job Award Agent } \\
\hline [g] Job Award Agent sends offer to other agents & \multirow[b]{2}{*}{ Job Award Agent } & & \multirow[b]{2}{*}{ Bid Evaluation Agent } \\
\hline according to the ranked bids & & & \\
\hline [h] Acknowledge of job offer & Job Award Agent & & Bid Evaluation Agent \\
\hline [i] Contract is made & Incoming Contract Agent & & Outgoing Contract Agent \\
\hline
\end{tabular}

Fig. 1. Simplified sequence of operations among agents.

complex scenarios where analytical solutions are not available. $\mathrm{Wu}[21]$ revealed that coordination mechanism in agent-based supply chains is a fundamental research area. In this connection, the research framework of this paper will base on agent-based coordination to solve inventory management problem in a distributed supply chain, subject to uncertain demand, by making use of early order completion contract.

\section{Simulation MODEL}

\section{A. Supply Chain Model}

The distributed supply chain in this study is modelled by multi-agent technique that was proposed in Chan and Chan [25]. Since this is not the main focus of this paper, only a simplified sequence diagram is shown in Fig. 1. Details of the agent-based model could be found in Chan and Chan [25].

The supply chain under study is a convergent type which consists of one retailer and four capacitated suppliers with single product type, in contrast to the divergent structure used in Zhao and Xie [12] and Zhao et al. [13], [14]. However, similar cost structure and demand pattern as presented in Zhao and Xie [12] and Zhao et al. [13], [14] will be used in this paper so that the simulation results on the demand uncertainty could be validated as compared with the trend of the simulation results of their studies. Although the model is simple, it is sufficient enough to be used for analysing the effects of demand uncertainty, and to demonstrate the usefulness of the proposed contract approach.

In the simulation study, actual demand is generated every period. One period can be interpreted as one day, one week, or even one month so that demand can always be grouped in a per period basis. Therefore, only the demand quantity per period will be varied in this study and hence the period is fixed. Similar to Zhao and Xie [12], and Zhao et al. [13], [14], retailer makes an order to the suppliers based on the Economic Order Quantity (EOQ) model, in which order quantity is given by (1), as follows:

$$
E O Q=\sqrt{\frac{2 k d}{h}}
$$

where $k$ is the ordering cost, $h$ is the Inventory holding cost per unit per period, and $d$ is the mean demand per period.

EOQ model is the best-known and most fundamental inventory model [26] but the model generally not taking uncertainty into consideration. In the EOQ model, demand is assumed as a constant value. This assumption is an ideal case that when no uncertainty exists in the system. By varying the demand per period, uncertainty of demand can be used as independent variables and the effects of the uncertainty can be quantified. Therefore, the EOQ model is used in order to demonstrate the effects of uncertain demand.

As discussed in Chan and Chan [25], the retailer announces an invitation for bids so that all suppliers are able to submit a proposal for the invitation. The suppliers will base on their own inventory and outstanding orders to come up with the proposed bids. Essentially, all suppliers can submit a bid but the lead time is different for different suppliers. Without loss of generality, transportation lead time is incorporated in the production lead time so that the former is assumed to be zero. This is a common assumption in the literature. After the retailer receives all the bids, the one with the shortest delivery due date will be selected. One may notice that this model is equivalent to considering the four homogenous suppliers as a single supplier with capacity equals to the sum of the capacity of all the four suppliers. To a certain extent this is true if the proposed early order completion contract is not taking into consideration. The reason that 
four suppliers are treated independently is because once a contract is completed earlier (decision model to be discussed later), the order cycle will be reset and hence the interaction is more complex than a single supplier, single buyer model. Therefore, four heterogeneous suppliers are assumed (and this assumption is more realistic in fact).

The simulation program is written in JAVA. Length of simulation is 465 periods while the first 100 periods are ignored for calculation in order to minimize the start-up effect. The final performance measures are based on the last 365 periods. If one period is equal to one day, then the effective length of simulation run is one year. Each simulation setting will be run with ten different random seeds and the average is reported in order to minimize the random effect.

\section{B. Cost Structure}

Total cost of the system is calculated for each simulation run. As mentioned above, the cost structure of this study is similar to the one presented in Zhao and Xie [12], and Zhao et al. [13], [14]. In fact, the effects of demand uncertainty that will be presented in the subsequent sections are in line with the findings of their studies. Therefore, the model in this study is supported indirectly. The total cost consists of several components which can be stated as follows.

- Product cost: it is charged per unit basis.

- Ordering cost: it is charged to the retailer whenever an order is placed by the retailer.

- Transportation cost: it is charged to the retailer whenever an order is sent to the retailer. In other words, it can be treated as another part of the ordering cost.

- Setup cost: it is charged to the supplier whenever an order is confirmed.

- Inventory holding cost: it is calculated per unit per day.

- Back order cost: it is calculated whenever inventory is not enough to cover demand and is calculated per unit per day.

\section{Demand Generation}

As mentioned in Section III-A, demand is generated in every period. The demand is calculated base on (2) that is similar to the one used in Zhao and Xie [12], and Zhao et al. [13], [14]:

$$
\begin{aligned}
\text { Demand }=\text { Base }+ & \text { Noise } \times \text { Normal } \\
=\text { Base }+ & \text { Demand Error }+ \text { Demand Variation } \\
& \times \text { Snormal })
\end{aligned}
$$

where Snormal is a random variable of standard normal.

In fact, more parameters have been included in Zhao and Xie [12], and Zhao et al. [13], [14] studies like demand with increasing and decreasing trends, with seasonality factors, and combinations of them. All these factors, including additional demand distributions other than normal distribution (like Poisson distribution), have been considered in this study as well. However, simulation results indicated that similar results could be achieved by employing the proposed early order completion contract approach. Therefore, the results are omitted here in order to simplify the presentation, and we could be more focused on the results of employing the coordination mechanism.
In this study, demand uncertainty is modelled as a Normal probability distribution. The mean and standard deviation of the function is equal to the demand error and demand variation in (2) respectively. The two parameters will be varied, i.e., they are the independent variables in this study. Demand error is assumed to be varied from $-30 \%$ of the base value to $+30 \%$ of the base value, at $10 \%$ interval (i.e., seven levels). Demand variation will be varied from $0 \%$ of the based value to $30 \%$ of the base value, also at $10 \%$ interval (i.e., four levels). In fact, more levels of uncertainties have been varied and tested but the results are consistent with different uncertainty levels. Therefore, simulation results against those levels mentioned above will be presented.

\section{Performance Measures}

In order to analyse the effects of demand uncertainty and the usefulness of the proposed coordination system, some suggested performance measures (i.e., dependent variables) are recorded as follows.

- Total system cost.

- Total management cost $=$ total system cost -total product cost.

- Total retailer cost.

- Total suppliers cost.

- Total inventory cost.

- Total back order cost.

- Fill rate of the whole system (i.e., fill rate of the retailer).

Traditional mathematical solutions (like the EOQ model) could optimize one of these performance measures only. Therefore, optimizing the total cost is usually the sole objective. However, in distributed supply chains (like the one in this simulation study), retailer and suppliers are independent units. Saving obtained from optimizing the total cost may not imply that all of them are better off. In fact, some of them are conflicting with each other (like the objective to minimize inventory cost and the objective to maximize fill rate). Therefore, individual performance measurements are also evaluated in this study.

\section{E. Details of Coordination Mechanism With Early Order Completion Contract}

If the demand of a supply chain is uncertain, the system is suffering from additional cost, and deterioration in fill rate. Particularly, when the demand error is positive, the inventory level would not be able to cover the required demand. Consequently, a large back order would be accumulated, which in turn induces a higher cost. In fact, this argument would be proven in the simulation results as shown in the following Section IV-A1 and Section IV-A2. In this connection, it is wise to implement a dynamic inventory replenishment policy to reduce the back order. In this study, a coordination mechanism is developed to reduce the impact of demand uncertainty by lowering the back order quantity, hence cost, and to improve the fill rate at zero demand error (details will be discussed later in Section IV). The procedures are highlighted as follows.

1) Instead of requesting a fixed quantity (i.e., the EOQ value) from the retailer as in the EOQ model, a contract is made 
between the retailer and supplier. In fact, it is a tuple which consists of a number of ordered pairs as shown in (3):
Contract $=\left\{\left(E O C Q_{1}, D_{1}\right), \ldots,\left(E O C Q_{i}, D_{i}\right) \ldots\right.$,

$$
\left.\left(E O C Q_{I}, D_{I}\right),\left(E O Q, D_{E O Q}\right)\right\} \text {. }
$$

A contract consists of a number of ordered pairs as shown in (3), while each ordered pair is composed of two parameters-the Early Order Completion Quantity (EOCQ) and the corresponding due date. They are indexed by $i$ (where $i \in 1$ to I). Without lost of generality, the ordered pairs are assumed to be arranged according to the due date (i.e., $\left.D_{1}<D_{2}<\ldots D_{i}<\ldots<D_{E O Q}\right)$. In this study, a linear structure of the contract is assumed and hence the due date could be calculated by (4):

$$
\begin{aligned}
D_{i}=D_{o}(\text { this_period }) & \\
& +\left(D_{E O Q}-D o(\text { this_period })\right) \times \frac{E O C Q_{i}}{E O Q}
\end{aligned}
$$

where $D_{o}(t)$ refers to period $t$ and hence $D_{o}$ (this_period) is the current period when the contract as in (3) is set up. In this connection, it is no need to change the invitation for bids and later decision models for both the suppliers and retailer as reflected in Fig. 1 if the coordination mechanism is employed. The only difference is that the set of quantity (i.e., $E O C Q_{1}, \ldots E O C Q_{I}, E O Q$ ) has to be included as they are part of the contract. These values, as calculated by (3) and (4), are determined once the contract is established. Please be reminded only $D_{i}$ values are related to the $E O C Q_{i}$ values, the $E O C Q_{i}$ values are not correlated so that they could be chosen by decision-makers according to different situations. For example, in a two ordered pairs contact, $E O C Q_{1}$ and $E O C Q_{2}$ may be set to $10 \%$ of $E O Q$ and $50 \%$ of $E O Q$ respectively. In fact, sensitivity analysis has been conducted on various settings, while some of them will be presented in Section IV.

Physical meaning of a particular ordered pair is that once $D_{i}$ reaches, the retailer is allowed to request the supplier to complete the contract by supplying $E O C Q_{i}$ to the retailer. If the supplier has enough inventories to fulfill the request (i.e., inventory larger than $E O C Q_{i}$ ), the supplier will ship that quantity to the retailer and the contract is ended. The $E O C Q_{i}$ is an important factor in this study which will be varied and be examined later. The objective is to determine when to complete the order ( $D_{E O Q}$ or any one of the $D_{i}$ ) and how many ( $E O Q$ or any one of the $E O C Q_{i}$ ) to be shipped subject to the inventory constraints. In addition, the simulation study will consider both single ordered pair and multiple ordered pairs as sensitivity analysis.

2) Specifically, when a period is elapsed, inventory of the retailer will be deducted according to the demand of that period by (2), and inventory of the suppliers will be increased according to their capacity. The retailer then calculates a variable called "inventory tightness" to reflect the uncertain situation, using (5). The variable is monitored continuously at the beginning of each period:

$$
\text { Inventory Tightness }=\frac{\text { Current Inventory }}{\text { Mean demand } \times d_{\text {next }}}
$$

where $d_{\text {next }}$ is the number of days to receive the next order as scheduled.

3) Two scenarios may be encountered as follows.

i) If the variable "inventory tightness" is higher than 1 , it is very likely that the inventory is enough to cover the actual demand before arrival of next order. No action will be enforced and the retailer will not send any message to the supplier, or vice versa. The flow will go back to step 2 for next period.

ii) On the other hand, if the variable is less than 1, the retailer will send a message (say "release early order") to the supplier to request for early order completion. The flow will continue to the next step 4.

4) Upon receipt of the request from the retailer, a supplier will check its contract and determine whether it has enough inventory to supply $E O C Q_{i}$, which corresponds to the ordered pair $\left(E O C Q_{i}, D_{i}\right)$ such that $D_{i} \leq D_{o}(t)<D_{i+1}$. Two possible actions are listed as follows.

i) If the inventory level of the supplier is less than the corresponding $E O C Q_{i}$, the supplier will send a reject message (say "reject") to the retailer.

ii) On the other hand, if the inventory level of that supplier is higher than the $E O C Q_{i}$, the supplier will send a accept message (say "ok") to the retailer.

5) Once the retailer collects all the replies from the suppliers, she will select the supplier who could supply the highest quantity, and then that quantity of inventory will be shipped to the retailer. Any costs that are incurred in this transaction are charged to the retailer. The ordering cycle will then be reset.

6) Above steps will be iterated and if there is no need to complete the order earlier, the order will be completed according to the traditional EOQ model at $D_{E O Q}$ eventually.

Effects of above coordination mechanism were evaluated through a set of simulation study, and the results will be presented in Section IV.

\section{Simulation Results}

As mentioned in Section III-C, there are two independent variables-demand error and demand variation. The former is modelled as seven levels and the latter is modelled as four levels. Therefore, there are $7 \times 4=28$ combinations for each simulation run. Important results are summarised below.

\section{A. Effects of Demand Uncertainty}

1) Effects of Demand Variation on Total System Cost: Fig. 2 shows the effect of demand variation on the percentage increase 


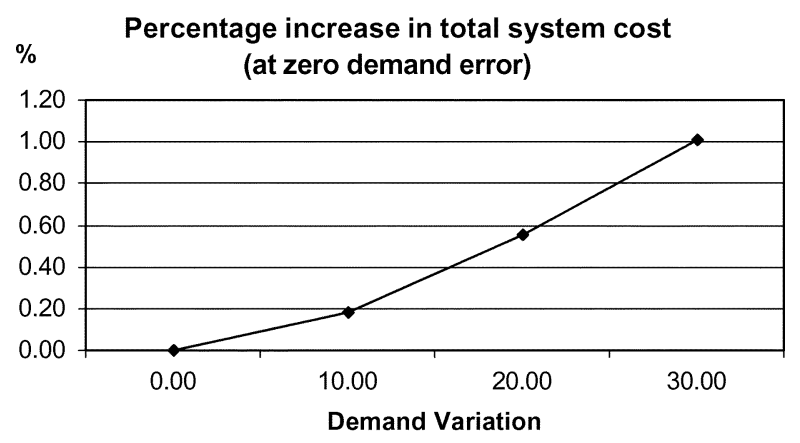

Fig. 2. Impact of demand variation on total system cost (at zero demand error).

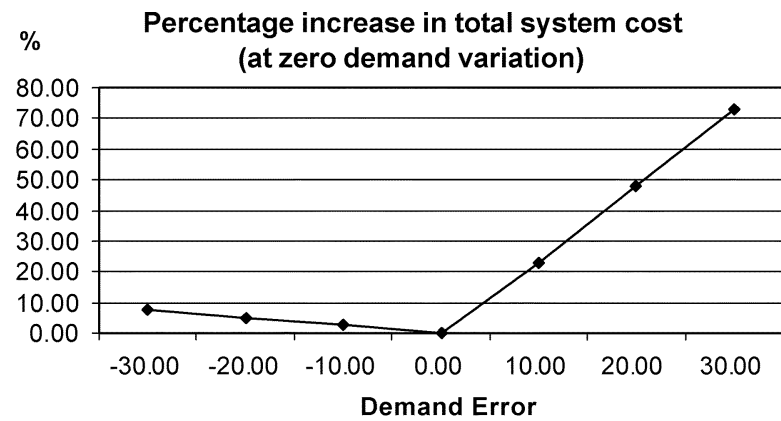

(a)

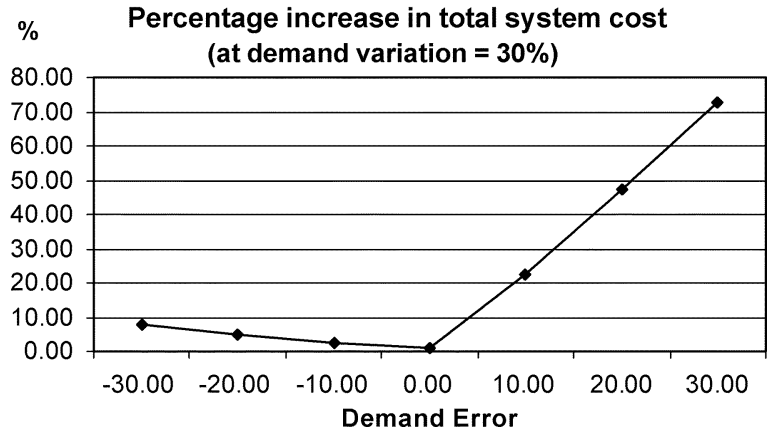

(b)

Fig. 3. Impact of demand error on total system cost. (a) Demand variation $=$ $0 \%$. (b) Demand variation $=30 \%$.

in the total system cost, at zero demand error. Please be reminded that the percentage increase in total system cost is reference to zero demand error and zero demand variation, which is an ideal situation without any uncertainty. An increasing trend is observed with increasing the magnitude of the demand variation. Only one setting is shown because similar trend can be observed for other settings (i.e., at various levels of demand error). Results of other settings are available on request through the correspondence author.

2) Effects of Demand Error on Total System Cost: Fig. 3 depicts the simulation results of percentage increase in total system cost against different demand errors of the system. Again, the percentage increase in total system cost is reference to zero demand error and zero demand variation. Although only the results when demand variations equal to zero [Fig. 3(a)] and $30 \%$ [Fig. 3(b)] are included, the trend is similar for different values of demand variation (i.e., $10 \%$ and $20 \%$ ) so that they are omitted for simplicity.
Obviously, the total system cost is increasing with the absolute value of the demand error. From both Fig. 3(a) and 3(b), the effect is more significant on the right hand side (i.e., positive demand error) than on the left hand side (i.e., negative demand error). Back order costs are the main contribution to the system cost increased on the right hand side, whereas inventory costs account for the system cost increased on the left hand side. In a long run, increasing suppliers' capacity, or equivalently, introducing more suppliers can reduce the effect of the system cost increased due to positive demand error. However, the additional cost due to this shift should be kept at minimal in a short run by adopting some remedy actions.

It can be concluded from Fig. 2 and Fig. 3 that both the demand error and demand variation affect the total system cost considerably, although the effect of demand error is of greater magnitude than the effect of demand variation. Therefore, the difference between Fig. 3(a) and Fig. 3(b) may not be visible. If the demand error and demand variation are treated as distorted information, Section IV-A1 and IV-A2 support the bullwhip effect indirectly as discussed by Lee et al. [16], and also the conclusion of Kimbrough et al. [23] that agents can play the beer game.

On top of these findings, similar analysis has been conducted on management cost, retailer costs, suppliers cost, inventory cost, backorder cost, and fill rate, etc. Since the trend of the results is similar and trivial, they are not presented here. In later section, only the improvement on the associated metrics by employing the proposed contract approach will be presented.

\section{B. Simulation Results With Coordination-With Single EOCQ Value}

Since the coordination mechanism as discussed in Section III has already pinpointed the impacts of positive demand error, the procedure will not be triggered if demand error is negative. Therefore, only the data obtained by varying positive demand error will be presented. As mentioned above, the EOCQ is a crucial parameter that will be varied. In fact, EOCQ will be set as a percentage of the quantity from EOQ model in this study. Different settings have been tested as sensitivity analysis and more than one EOCQ values (i.e., I > 1 as in the contract) have been studied. Selected representative results in terms of percentage improvement of the performance measures will be presented in the following sub-sections. In this sub-section (Section IV-B), results with one ordered pair (i.e., $\mathrm{I}=1$ ) in the contract will be evaluated first. Since only one EOCQ value gets involved, the subscript $i$ in this sub-section is omitted. In Section IV-C, results with multiple EOCQ values (i.e., I $>1$ ) will be presented.

1) Total Unit Cost and Management Cost: Instead of comparing the actual total cost, Table I shows the percentage improvement of using the coordination mechanism with different EOCQ values. Negative value means improvement (i.e., cost decrease), while positive value means cost is increased. Same definition will be applied in the following sections, except Section IV-B4. Improved values are highlighted (bold and italic) as well to improve readability. Unit cost is used because the total output quantity is different after the coordination mechanism is 
TABLE I

PERCENTAGE IMPROVEMENT IN TOTAL UNIT COST

\begin{tabular}{|c|c|c|c|c|}
\cline { 2 - 5 } \multicolumn{1}{c|}{} & \multicolumn{4}{c|}{ Demand Variation } \\
\hline Demand Error & $0 \%$ & $10 \%$ & $20 \%$ & $30 \%$ \\
\hline $0 \%$ & 0.00 & 1.81 & 2.16 & 2.22 \\
\hline $10 \%$ & 4.62 & 4.62 & 4.63 & 4.63 \\
\hline $20 \%$ & 3.47 & 3.48 & 3.49 & 3.49 \\
\hline $30 \%$ & 2.66 & 2.67 & 2.67 & 2.67 \\
\hline
\end{tabular}

(i) EOCQ value $=20 \%$ of EOQ

\begin{tabular}{|c|c|c|c|c|}
\cline { 2 - 5 } \multicolumn{1}{c|}{} & \multicolumn{4}{c|}{ Demand Variation } \\
\hline Demand Error & $0 \%$ & $10 \%$ & $20 \%$ & $30 \%$ \\
\hline $0 \%$ & 0.00 & 1.14 & 1.07 & 0.90 \\
\hline $10 \%$ & $-\mathbf{1 . 1 9}$ & $-\mathbf{1 . 1 7}$ & -1.14 & -1.10 \\
\hline $20 \%$ & -1.35 & $-\mathbf{1 . 3 5}$ & $-\mathbf{1 . 3 5}$ & $-\mathbf{1 . 3 5}$ \\
\hline $30 \%$ & $-\mathbf{1 . 4 6}$ & $-\mathbf{1 . 4 6}$ & $-\mathbf{1 . 4 6}$ & $-\mathbf{1 . 4 6}$ \\
\hline
\end{tabular}

(ii) $\mathrm{EOCQ}$ value $=40 \%$ of $\mathrm{EOQ}$

\begin{tabular}{|c|c|c|c|c|}
\cline { 2 - 5 } \multicolumn{1}{c|}{} & \multicolumn{4}{c|}{ Demand Variation } \\
\hline Demand Error & $0 \%$ & $10 \%$ & $20 \%$ & $30 \%$ \\
\hline $0 \%$ & 0.00 & 1.12 & 0.88 & 0.66 \\
\hline $10 \%$ & $\mathbf{- 0 . 8 5}$ & $\mathbf{- 0 . 8 5}$ & $\mathbf{- 0 . 8 4}$ & $\mathbf{- 0 . 8 2}$ \\
\hline $20 \%$ & $\mathbf{- 0 . 9 4}$ & $\mathbf{- 0 . 9 4}$ & $\mathbf{- 0 . 9 4}$ & $\mathbf{- 0 . 9 4}$ \\
\hline $30 \%$ & $\mathbf{- 1 . 0 0}$ & $\mathbf{- 1 . 0 0}$ & $\mathbf{- 1 . 0 0}$ & $\mathbf{- 1 . 0 0}$ \\
\hline
\end{tabular}

(iii)EOCQ value $=50 \%$ of $\mathrm{EOQ}$

\begin{tabular}{|c|c|c|c|c|}
\cline { 2 - 5 } \multicolumn{1}{c|}{} & \multicolumn{4}{c|}{ Demand Variation } \\
\hline Demand Error & $0 \%$ & $10 \%$ & $20 \%$ & $30 \%$ \\
\hline $0 \%$ & 0.00 & 1.11 & 0.78 & 0.48 \\
\hline $10 \%$ & $\mathbf{- 2 . 0 7}$ & $\mathbf{- 2 . 0 7}$ & -2.05 & $-\mathbf{2 . 0 2}$ \\
\hline $20 \%$ & $\mathbf{- 1 . 9 9}$ & $\mathbf{- 1 . 9 9}$ & $-\mathbf{1 . 9 9}$ & $-\mathbf{1 . 9 9}$ \\
\hline $30 \%$ & $\mathbf{- 1 . 9 4}$ & $\mathbf{- 1 . 9 4}$ & $\mathbf{- 1 . 9 4}$ & $\mathbf{- 1 . 9 4}$ \\
\hline
\end{tabular}

(iv) $\mathrm{EOCQ}$ value $=60 \%$ of $\mathrm{EOQ}$

\begin{tabular}{|c|c|c|c|c|}
\cline { 2 - 5 } \multicolumn{1}{c|}{} & \multicolumn{4}{c|}{ Demand Variation } \\
\hline Demand Error & $0 \%$ & $10 \%$ & $20 \%$ & $30 \%$ \\
\hline $0 \%$ & 0.00 & 1.10 & 0.83 & 0.63 \\
\hline $10 \%$ & $\mathbf{- 0 . 6 6}$ & $\mathbf{- 0 . 6 6}$ & $\mathbf{- 0 . 6 6}$ & $\mathbf{- 0 . 6 4}$ \\
\hline $20 \%$ & $\mathbf{- 0 . 7 4}$ & $\mathbf{- 0 . 7 3}$ & $\mathbf{- 0 . 7 3}$ & $\mathbf{- 0 . 7 3}$ \\
\hline $30 \%$ & $\mathbf{- 0 . 7 9}$ & $\mathbf{- 0 . 7 9}$ & $\mathbf{- 0 . 7 9}$ & $\mathbf{- 0 . 7 8}$ \\
\hline
\end{tabular}

(v) EOCQ value $=80 \%$ of EOQ

imposed. Therefore, it is only fair to compare different situations by the unit cost.

Results indicate that if the EOCQ value is sufficiently large (greater than $20 \%$ of EOQ values in this study), unit cost would be decreased when demand error is nonzero. This is because whenever the rule is triggered, part of the inventory from suppliers will be shipped to the retailers to recover any back order immediately. This action leads to reduction in supplier inventory and retailer back order, and hence the total system cost. When the EOCQ is set at $60 \%$ of the EOQ quantity, the improvement is the best among the settings under evaluation. In fact, this is related to a dilemma that when the rule is triggered, back order cost can be reduced while additional management costs are incurred. Therefore, if the EOCQ value is too low, the additional management cost cannot be compensated by the reduction in back order cost.

Similar to the total unit cost, improvement in unit management cost is reported in Table II instead of the actual value. Similar findings from the total unit cost can be observed in the management cost. Again, the best improvement among the EOCQ value is set at $60 \%$ of EOQ quantity. In fact, this measure is a better index than the total unit cost to reflect the actual improvement of the proposed rule. It is because the product cost, which is the largest portion of cost, is not involved in the management
TABLE II

PERCENTAGE IMPROVEMENT IN TOTAL UnIT COST (a) EOCQ value = $20 \%$ of $\mathrm{EOQ}$; (b) $\mathrm{EOCQ}$ value $=40 \%$ of $\mathrm{EOQ}$; (c)

$\mathrm{EOCQ}$ value $=50 \%$ of $\mathrm{EOQ} ;$ (d) $\mathrm{EOCQ}$ value $=60 \%$ of $\mathrm{EOQ}$; (e) $\mathrm{EOCQ}$ value $=80 \%$ of $\mathrm{EOQ}$

\begin{tabular}{|c|c|c|c|c|}
\cline { 2 - 5 } \multicolumn{1}{c|}{} & \multicolumn{4}{c|}{ Demand Variation } \\
\hline Demand Error & $0 \%$ & $10 \%$ & $20 \%$ & $30 \%$ \\
\hline $0 \%$ & 0.00 & 42.56 & 46.94 & 44.25 \\
\hline $10 \%$ & 21.03 & 21.15 & 21.27 & 21.38 \\
\hline $20 \%$ & 9.88 & 9.92 & 9.96 & 9.99 \\
\hline $30 \%$ & 5.97 & 5.99 & 6.01 & 6.03 \\
\hline
\end{tabular}

(a)

\begin{tabular}{|c|c|c|c|c|}
\cline { 2 - 5 } \multicolumn{1}{c|}{} & \multicolumn{4}{c|}{ Demand Variation } \\
\hline Demand Error & $0 \%$ & $10 \%$ & $20 \%$ & $30 \%$ \\
\hline $0 \%$ & 0.00 & 26.91 & 23.29 & 17.86 \\
\hline $10 \%$ & $\mathbf{- 5 . 4 0}$ & $\mathbf{- 5 . 3 8}$ & $\mathbf{- 5 . 2 5}$ & $\mathbf{- 5 . 1 0}$ \\
\hline $20 \%$ & $\mathbf{- 3 . 8 4}$ & $\mathbf{- 3 . 8 4}$ & $\mathbf{- 3 . 8 5}$ & $\mathbf{- 3 . 8 6}$ \\
\hline $30 \%$ & $\mathbf{- 3 . 2 9}$ & $\mathbf{- 3 . 2 9}$ & $\mathbf{- 3 . 2 9}$ & $\mathbf{- 3 . 3 0}$ \\
\hline
\end{tabular}

(b)

\begin{tabular}{|c|c|c|c|c|}
\cline { 2 - 5 } \multicolumn{1}{c|}{} & \multicolumn{4}{c|}{ Demand Variation } \\
\hline Demand Error & $0 \%$ & $10 \%$ & $20 \%$ & $30 \%$ \\
\hline $0 \%$ & 0.00 & 26.41 & 19.12 & 13.22 \\
\hline $10 \%$ & $\mathbf{- 3 . 8 8}$ & $\mathbf{- 3 . 8 8}$ & $\mathbf{- 3 . 8 6}$ & $\mathbf{- 3 . 7 7}$ \\
\hline $20 \%$ & $\mathbf{- 2 . 6 6}$ & $\mathbf{- 2 . 6 7}$ & $\mathbf{- 2 . 6 7}$ & $\mathbf{- 2 . 6 8}$ \\
\hline $30 \%$ & $\mathbf{- 2 . 2 4}$ & $\mathbf{- 2 . 2 4}$ & $\mathbf{- 2 . 2 4}$ & $\mathbf{- 2 . 2 4}$ \\
\hline
\end{tabular}

(c)

\begin{tabular}{|c|c|c|c|c|}
\cline { 2 - 5 } \multicolumn{1}{c|}{} & \multicolumn{4}{c|}{ Demand Variation } \\
\hline Demand Error & $0 \%$ & $10 \%$ & $20 \%$ & $30 \%$ \\
\hline $0 \%$ & 0.00 & 26.24 & 17.03 & 9.50 \\
\hline $10 \%$ & $\mathbf{- 9 . 4 1}$ & $\mathbf{- 9 . 4 5}$ & $\mathbf{- 9 . 4 4}$ & $\mathbf{- 9 . 3 2}$ \\
\hline $20 \%$ & $\mathbf{- 5 . 6 6}$ & $\mathbf{- 5 . 6 7}$ & $\mathbf{- 5 . 6 9}$ & $\mathbf{- 5 . 7 0}$ \\
\hline $30 \%$ & $\mathbf{- 4 . 3 5}$ & $\mathbf{- 4 . 3 5}$ & $\mathbf{- 4 . 3 6}$ & $\mathbf{- 4 . 3 7}$ \\
\hline
\end{tabular}

(d)

\begin{tabular}{|c|c|c|c|c|}
\cline { 2 - 5 } \multicolumn{1}{c|}{} & \multicolumn{4}{c|}{ Demand Variation } \\
\hline Demand Error & $0 \%$ & $10 \%$ & $20 \%$ & $30 \%$ \\
\hline $0 \%$ & 0.00 & 25.94 & 18.14 & 12.54 \\
\hline $10 \%$ & $\mathbf{- 3 . 0 2}$ & $\mathbf{- 3 . 0 4}$ & $\mathbf{- 3 . 0 3}$ & $\mathbf{- 2 . 9 5}$ \\
\hline $20 \%$ & $\mathbf{- 2 . 0 9}$ & $\mathbf{- 2 . 0 9}$ & $\mathbf{- 2 . 1 0}$ & $\mathbf{- 2 . 1 0}$ \\
\hline $30 \%$ & $\mathbf{- 1 . 7 6}$ & $\mathbf{- 1 . 7 7}$ & $\mathbf{- 1 . 7 7}$ & $\mathbf{- 1 . 7 7}$ \\
\hline
\end{tabular}

(e)

cost. In Table II(d), it can be observed that the maximum improvement can be found is more than $9 \%$ when demand error is $10 \%$.

Another observation can be drawn from both Tables I and II is that cost is increased regardless of the demand variation and EOCQ value, when demand error is zero. This is understandable because when the demand error is zero, the absolute back order quantity would not be too high. As mentioned above, the proposed coordination mechanism will add extra management cost but the reduction in back order cost is not significant in this case to compensate the extra cost. It seems that the proposed coordination mechanism is not applicable when demand error is small. However, another improvement can be found in fill rate at zero demand error. This will be discussed in Section IV-B4.

2) Retailer Cost and Suppliers Cost: With respect to retailer cost, similar improvement can be found if the EOCQ value is sufficiently large, and the best improvement can also be found when the value is set at $60 \%$ of the EOQ quantity. In fact, similar findings and discussions can be applied to the retailer cost as stated in Section IV-B1. Therefore, the numerical results of retailer are omitted here. 
TABLE III

PERCENTAGE IMPROVEMENT IN TOTAL UNIT SUPPLIERS COST. (a) $\mathrm{EOCQ}$ value $=20 \%$ of $\mathrm{EOQ}$; (b) $\mathrm{EOCQ}$ value $=40 \%$ of $\mathrm{EOQ}$ (c) $\mathrm{EOCQ}$ value $=50 \%$ of $\mathrm{EOQ}$; (d) $\mathrm{EOCQ}$ value $=60 \%$ of $\mathrm{EOQ}$; (e) $\mathrm{EOCQ}$ value $=80 \%$ of $\mathrm{EOQ}$

\begin{tabular}{|c|c|c|c|c|}
\cline { 2 - 5 } \multicolumn{1}{c|}{} & \multicolumn{4}{c|}{ Demand Variation } \\
\hline Demand Error & $0 \%$ & $10 \%$ & $20 \%$ & $30 \%$ \\
\hline $0 \%$ & 0.00 & $\mathbf{- 2 . 5 7}$ & $\mathbf{- 4 . 0 6}$ & $\mathbf{- 5 . 4 2}$ \\
\hline $10 \%$ & $\mathbf{- 3 0 . 1 6}$ & $\mathbf{- 3 0 . 1 4}$ & $\mathbf{- 3 0 . 0 3}$ & $\mathbf{- 2 9 . 8 3}$ \\
\hline $20 \%$ & $\mathbf{- 3 0 . 1 6}$ & $\mathbf{- 3 0 . 1 6}$ & $\mathbf{- 3 0 . 1 6}$ & $\mathbf{- 3 0 . 1 6}$ \\
\hline $30 \%$ & $\mathbf{- 3 0 . 1 6}$ & $\mathbf{- 3 0 . 1 6}$ & $\mathbf{- 3 0 . 1 6}$ & $\mathbf{- 3 0 . 1 6}$ \\
\hline
\end{tabular}

(a)

\begin{tabular}{|c|c|c|c|c|}
\cline { 2 - 5 } \multicolumn{1}{c|}{} & \multicolumn{4}{c|}{ Demand Variation } \\
\hline Demand Error & $0 \%$ & $10 \%$ & $20 \%$ & $30 \%$ \\
\hline $0 \%$ & 0.00 & $\mathbf{- 3 . 7 3}$ & $\mathbf{- 5 . 2 6}$ & $\mathbf{- 6 . 3 4}$ \\
\hline $10 \%$ & $\mathbf{- 2 5 . 1 4}$ & $\mathbf{- 2 5 . 1 4}$ & $\mathbf{- 2 5 . 1 0}$ & $\mathbf{- 2 5 . 0 0}$ \\
\hline $20 \%$ & $\mathbf{- 2 5 . 1 4}$ & $\mathbf{- 2 5 . 1 4}$ & $\mathbf{- 2 5 . 1 4}$ & $\mathbf{- 2 5 . 1 4}$ \\
\hline $30 \%$ & $\mathbf{- 2 5 . 1 4}$ & $\mathbf{- 2 5 . 1 4}$ & $\mathbf{- 2 5 . 1 4}$ & $\mathbf{- 2 5 . 1 4}$ \\
\hline
\end{tabular}

(b)

\begin{tabular}{|c|c|c|c|c|}
\cline { 2 - 5 } \multicolumn{1}{c|}{} & \multicolumn{4}{c|}{ Demand Variation } \\
\hline Demand Error & $0 \%$ & $10 \%$ & $20 \%$ & $30 \%$ \\
\hline $0 \%$ & 0.00 & $\mathbf{- 4 . 3 2}$ & $\mathbf{- 5 . 4 8}$ & $\mathbf{- 5 . 9 3}$ \\
\hline $10 \%$ & $\mathbf{- 1 9 . 1 0}$ & $\mathbf{- 1 9 . 1 0}$ & $\mathbf{- 1 9 . 0 9}$ & $\mathbf{- 1 9 . 0 7}$ \\
\hline $20 \%$ & $\mathbf{- 1 9 . 1 0}$ & $\mathbf{- 1 9 . 1 0}$ & $\mathbf{- 1 9 . 1 0}$ & $\mathbf{- 1 9 . 1 0}$ \\
\hline $30 \%$ & $\mathbf{- 1 9 . 1 0}$ & $\mathbf{- 1 9 . 1 0}$ & $\mathbf{- 1 9 . 1 0}$ & $\mathbf{- 1 9 . 1 0}$ \\
\hline
\end{tabular}

(c)

\begin{tabular}{|c|c|c|c|c|}
\cline { 2 - 5 } \multicolumn{1}{c|}{} & \multicolumn{4}{c|}{ Demand Variation } \\
\hline Demand Error & $0 \%$ & $10 \%$ & $20 \%$ & $30 \%$ \\
\hline $0 \%$ & 0.00 & -4.91 & $\mathbf{- 5 . 9 1}$ & $\mathbf{- 6 . 2 9}$ \\
\hline $10 \%$ & $\mathbf{- 1 9 . 0 1}$ & $\mathbf{- 1 9 . 0 1}$ & $\mathbf{- 1 9 . 0 1}$ & $\mathbf{- 1 8 . 9 9}$ \\
\hline $20 \%$ & $\mathbf{- 1 9 . 0 1}$ & $\mathbf{- 1 9 . 0 1}$ & $\mathbf{- 1 9 . 0 1}$ & $\mathbf{- 1 9 . 0 1}$ \\
\hline $30 \%$ & $\mathbf{- 1 9 . 0 1}$ & $\mathbf{- 1 9 . 0 1}$ & $\mathbf{- 1 9 . 0 1}$ & $\mathbf{- 1 9 . 0 1}$ \\
\hline
\end{tabular}

(d)

\begin{tabular}{|c|c|c|c|c|}
\cline { 2 - 5 } \multicolumn{1}{c|}{} & \multicolumn{4}{c|}{ Demand Variation } \\
\hline Demand Error & $0 \%$ & $10 \%$ & $20 \%$ & $30 \%$ \\
\hline $0 \%$ & 0.00 & $\mathbf{- 6 . 0 5}$ & $\mathbf{- 6 . 0 8}$ & $\mathbf{- 6 . 0 6}$ \\
\hline $10 \%$ & $\mathbf{- 1 4 . 0 6}$ & $\mathbf{- 1 4 . 0 6}$ & $\mathbf{- 1 4 . 0 6}$ & $\mathbf{- 1 4 . 0 6}$ \\
\hline $20 \%$ & $\mathbf{- 1 4 . 0 6}$ & $\mathbf{- 1 4 . 0 6}$ & -14.06 & -14.06 \\
\hline $30 \%$ & $\mathbf{- 1 4 . 0 6}$ & $\mathbf{- 1 4 . 0 6}$ & $\mathbf{- 1 4 . 0 6}$ & $\mathbf{- 1 4 . 0 6}$ \\
\hline
\end{tabular}

(e)

Table III shows the improvement in suppliers cost. The results are not the same as the total unit cost, management cost, or retailer cost as reported previously. Improvement can be found regardless of the demand error and variation, and EOCQ value. This is because whenever the rule is triggered, part of the supplier inventory will be reduced and hence the overall suppliers cost is decreased. The higher the frequency to trigger the rule, the higher is the saving. Hence, the best improvement is found when the EOCQ value is set at $20 \%$ of the EOQ quantity.

3) Inventory Cost and Back Order Cost: Results of inventory cost are similar to the supplier cost as discussed in Section IV-B2. This is because the inventory cost is related to the suppliers' side when back order is encountered - the retailer has no or little inventory in such situation. Therefore, numerical results and discussion are not repeated for inventory cost.

Improvement in back order cost is summarised in Table IV. Since the proposed contract approach aims at reducing back order quantity, and hence back order cost, percentage improvement can be found regardless of the demand error and deviation,
TABLE IV

PERCENTAGE IMPROVEMENT IN TOTAL UNIT BACK ORDER COST (a) $\mathrm{EOCQ}$ value $=20 \%$ of $\mathrm{EOQ}$; (b) $\mathrm{EOCQ}$ value $=40 \%$ of $\mathrm{EOQ}$; (c) $\mathrm{EOCQ}$ value $=50 \%$ of $\mathrm{EOQ}$; (d) $\mathrm{EOCQ}$ value $=60 \%$ of $\mathrm{EOQ}$; (e) $\mathrm{EOCQ}$ value $=80 \%$ of $\mathrm{EOQ}$

\begin{tabular}{|c|c|c|c|c|}
\cline { 2 - 5 } \multicolumn{1}{c|}{} & \multicolumn{4}{c|}{ Demand Variation } \\
\hline Demand Error & $0 \%$ & $10 \%$ & $20 \%$ & $30 \%$ \\
\hline $0 \%$ & N/A & $\mathbf{- 9 9 . 6 6}$ & $\mathbf{- 9 9 . 4 3}$ & $\mathbf{- 9 7 . 3 1}$ \\
\hline $10 \%$ & $\mathbf{- 3 3 . 5 4}$ & $\mathbf{- 3 3 . 7 4}$ & $\mathbf{- 3 3 . 8 0}$ & $\mathbf{- 3 3 . 7 1}$ \\
\hline $20 \%$ & $\mathbf{- 1 7 . 2 2}$ & $\mathbf{- 1 7 . 2 7}$ & $\mathbf{- 1 7 . 3 2}$ & $\mathbf{- 1 7 . 3 7}$ \\
\hline $30 \%$ & $\mathbf{- 1 2 . 0 6}$ & $\mathbf{- 1 2 . 0 8}$ & $\mathbf{- 1 2 . 1 1}$ & $\mathbf{- 1 2 . 1 3}$ \\
\hline
\end{tabular}

(a)

\begin{tabular}{|c|c|c|c|c|}
\cline { 2 - 5 } \multicolumn{1}{c|}{} & \multicolumn{4}{c|}{ Demand Variation } \\
\hline Demand Error & $0 \%$ & $10 \%$ & $20 \%$ & $30 \%$ \\
\hline $0 \%$ & N/A & $\mathbf{- 9 9 . 8 3}$ & $\mathbf{- 9 9 . 7 2}$ & $\mathbf{- 9 2 . 6 4}$ \\
\hline $10 \%$ & $\mathbf{- 2 7 . 9 2}$ & $\mathbf{- 2 8 . 0 6}$ & $\mathbf{- 2 8 . 1 0}$ & $\mathbf{- 2 8 . 0 4}$ \\
\hline $20 \%$ & $\mathbf{- 1 4 . 5 2}$ & $\mathbf{- 1 4 . 5 7}$ & $\mathbf{- 1 4 . 6 1}$ & $\mathbf{- 1 4 . 6 5}$ \\
\hline $30 \%$ & $\mathbf{- 1 0 . 2 9}$ & $\mathbf{- 1 0 . 3 1}$ & $\mathbf{- 1 0 . 3 3}$ & $\mathbf{- 1 0 . 3 5}$ \\
\hline
\end{tabular}

(b)

\begin{tabular}{|c|c|c|c|c|}
\cline { 2 - 5 } \multicolumn{1}{c|}{} & \multicolumn{4}{c|}{ Demand Variation } \\
\hline Demand Error & $0 \%$ & $10 \%$ & $20 \%$ & $30 \%$ \\
\hline $0 \%$ & N/A & $\mathbf{- 9 9 . 8 3}$ & $\mathbf{- 9 5 . 4 7}$ & $\mathbf{- 8 1 . 5 0}$ \\
\hline $10 \%$ & $\mathbf{- 2 0 . 7 0}$ & $\mathbf{- 2 0 . 8 2}$ & $\mathbf{- 2 0 . 9 0}$ & $\mathbf{- 2 0 . 8 7}$ \\
\hline $20 \%$ & $\mathbf{- 1 0 . 6 4}$ & $\mathbf{- 1 0 . 6 7}$ & $\mathbf{- 9 1 . 0 6}$ & $\mathbf{- 1 0 . 7 4}$ \\
\hline $30 \%$ & $\mathbf{- 7 . 4 6}$ & $\mathbf{- 7 . 4 8}$ & $\mathbf{- 7 . 4 9}$ & $\mathbf{- 7 . 5 0}$ \\
\hline
\end{tabular}

(c)

\begin{tabular}{|c|c|c|c|c|}
\cline { 2 - 5 } \multicolumn{1}{c|}{} & \multicolumn{4}{c|}{ Demand Variation } \\
\hline Demand Error & $0 \%$ & $10 \%$ & $20 \%$ & $30 \%$ \\
\hline $0 \%$ & N/A & $\mathbf{- 9 9 . 8 2}$ & $\mathbf{- 9 7 . 0 9}$ & $\mathbf{- 8 3 . 1 7}$ \\
\hline $10 \%$ & $\mathbf{- 2 1 . 1 1}$ & $\mathbf{- 2 1 . 2 4}$ & $\mathbf{- 2 1 . 3 2}$ & $\mathbf{- 2 1 . 2 9}$ \\
\hline $20 \%$ & $\mathbf{- 1 0 . 9 8}$ & $\mathbf{- 1 1 . 0 1}$ & $\mathbf{- 1 1 . 0 4}$ & $\mathbf{- 1 1 . 0 7}$ \\
\hline $30 \%$ & $\mathbf{- 7 . 7 7}$ & $\mathbf{- 7 . 7 9}$ & $\mathbf{- 7 . 8 0}$ & $\mathbf{- 7 . 8 2}$ \\
\hline
\end{tabular}

(d)

\begin{tabular}{|c|c|c|c|c|}
\cline { 2 - 5 } \multicolumn{1}{c|}{} & \multicolumn{4}{c|}{ Demand Variation } \\
\hline Demand Error & $0 \%$ & $10 \%$ & $20 \%$ & $30 \%$ \\
\hline $0 \%$ & N/A & $\mathbf{- 9 9 . 8 3}$ & $\mathbf{- 8 4 . 6 2}$ & $\mathbf{- 6 5 . 7 1}$ \\
\hline $10 \%$ & $\mathbf{- 1 4 . 1 7}$ & $\mathbf{- 1 4 . 2 7}$ & $\mathbf{- 1 4 . 3 4}$ & $\mathbf{- 1 4 . 3 5}$ \\
\hline $20 \%$ & $\mathbf{- 7 . 3 7}$ & $\mathbf{- 7 . 3 9}$ & $\mathbf{- 7 . 4 1}$ & $\mathbf{- 7 . 4 3}$ \\
\hline $30 \%$ & $\mathbf{- 5 . 2 2}$ & $\mathbf{- 5 . 2 3}$ & $\mathbf{- 5 . 2 4}$ & $\mathbf{- 5 . 2 5}$ \\
\hline
\end{tabular}

(e)

and the EOCQ value. Again, the smaller the EOCQ value, the higher the back order cost can be reduced because back order can be eliminated in a faster pace. Therefore, maximum improvement can be found when the EOCQ value is set at $20 \%$ of the EOQ quantity.

4) Fill Rate: The results of the fill rate are quite different from the above performance measures and they are listed in Table V. First of all, improvement is presented as positive value since the higher the fill rate, the better is the performance. As mentioned before, when demand error is too large, the system is unable to recover the back order quantity. Therefore, no improvement can be found when demand error is more than $10 \%$. However, the proposed contract approach would not worsen the performance of the system in terms of fill rate. Nevertheless, attention should be paid on zero demand error. Improvement can be found at any EOCQ values when the demand error is zero. That means at zero demand error, the proposed contract approach succeeded in improving the fill rate. Fig. 4 illustrates the fill rate at different EOCQ values. 
TABLE V

PPERCENTAGe IMPROVEMENT IN Fill RATE. (a) EOCQ value $=$ $20 \%$ of EOQ; (b) EOCQ value $=40 \%$ of $\mathrm{EOQ}$

(c) $\mathrm{EOCQ}$ value $=50 \%$ of $\mathrm{EOQ}$; (d) $\mathrm{EOCQ}$ value $=60 \%$ of $\mathrm{EOQ}$; (e) $\mathrm{EOCQ}$ value $=80 \%$ of $\mathrm{EOQ}$

\begin{tabular}{|c|c|c|c|c|}
\cline { 2 - 5 } \multicolumn{1}{c|}{} & \multicolumn{4}{c|}{ Demand Variation } \\
\hline Demand Error & $0 \%$ & $10 \%$ & $20 \%$ & $30 \%$ \\
\hline $0 \%$ & 0.00 & $\mathbf{2 . 8 2}$ & $\mathbf{4 . 6 0}$ & $\mathbf{4 . 9 3}$ \\
\hline $10 \%$ & 0.00 & 0.00 & $\mathbf{0 . 3 4}$ & $\mathbf{1 . 0 3}$ \\
\hline $20 \%$ & 0.00 & 0.00 & 0.00 & 0.00 \\
\hline $30 \%$ & 0.00 & 0.00 & 0.00 & 0.00 \\
\hline
\end{tabular}

(a)

\begin{tabular}{|c|c|c|c|c|}
\cline { 2 - 5 } \multicolumn{1}{c|}{} & \multicolumn{4}{c|}{ Demand Variation } \\
\hline Demand Error & $0 \%$ & $10 \%$ & $20 \%$ & $30 \%$ \\
\hline $0 \%$ & 0.00 & $\mathbf{2 . 8 2}$ & $\mathbf{4 . 6 0}$ & 4.64 \\
\hline $10 \%$ & 0.00 & $\mathbf{0 . 3 4}$ & $\mathbf{0 . 6 8}$ & $\mathbf{1 . 0 3}$ \\
\hline $20 \%$ & 0.00 & 0.00 & 0.00 & 0.00 \\
\hline $30 \%$ & 0.00 & 0.00 & 0.00 & 0.00 \\
\hline
\end{tabular}

(b)

\begin{tabular}{|c|c|c|c|c|}
\cline { 2 - 5 } \multicolumn{1}{c|}{} & \multicolumn{4}{c|}{ Demand Variation } \\
\hline Demand Error & $0 \%$ & $10 \%$ & $20 \%$ & $30 \%$ \\
\hline $0 \% 0.00$ & & $\mathbf{2 . 8 2}$ & 4.31 & 4.06 \\
\hline $10 \%$ & 0.00 & 0.00 & $\mathbf{0 . 3 4}$ & $\mathbf{0 . 6 8}$ \\
\hline $20 \%$ & 0.00 & 0.00 & 0.00 & 0.00 \\
\hline $30 \%$ & 0.00 & 0.00 & 0.00 & 0.00 \\
\hline
\end{tabular}

(c)

\begin{tabular}{|c|c|c|c|c|}
\cline { 2 - 5 } \multicolumn{1}{c|}{} & \multicolumn{4}{c|}{ Demand Variation } \\
\hline Demand Error & $0 \%$ & $10 \%$ & $20 \%$ & $30 \%$ \\
\hline $0 \%$ & 0.00 & $\mathbf{2 . 8 2}$ & $\mathbf{4 . 3 1}$ & 4.06 \\
\hline $10 \%$ & 0.00 & 0.00 & $\mathbf{0 . 3 4}$ & $\mathbf{0 . 6 8}$ \\
\hline $20 \%$ & 0.00 & 0.00 & 0.00 & 0.00 \\
\hline $30 \%$ & 0.00 & 0.00 & 0.00 & 0.00 \\
\hline
\end{tabular}

(d)

\begin{tabular}{|c|c|c|c|c|}
\cline { 2 - 5 } \multicolumn{1}{c|}{} & \multicolumn{4}{c|}{ Demand Variation } \\
\hline Demand Error & $0 \%$ & $10 \%$ & $20 \%$ & $30 \%$ \\
\hline $0 \%$ & 0.00 & $\mathbf{2 . 8 2}$ & $\mathbf{3 . 4 5}$ & $\mathbf{3 . 1 9}$ \\
\hline $10 \%$ & 0.00 & 0.00 & $\mathbf{0 . 3 4}$ & 0.00 \\
\hline $20 \%$ & 0.00 & 0.00 & 0.00 & 0.00 \\
\hline $30 \%$ & 0.00 & 0.00 & 0.00 & 0.00 \\
\hline
\end{tabular}

(e)

Fill Rate

$\%$ (at zero demand error)

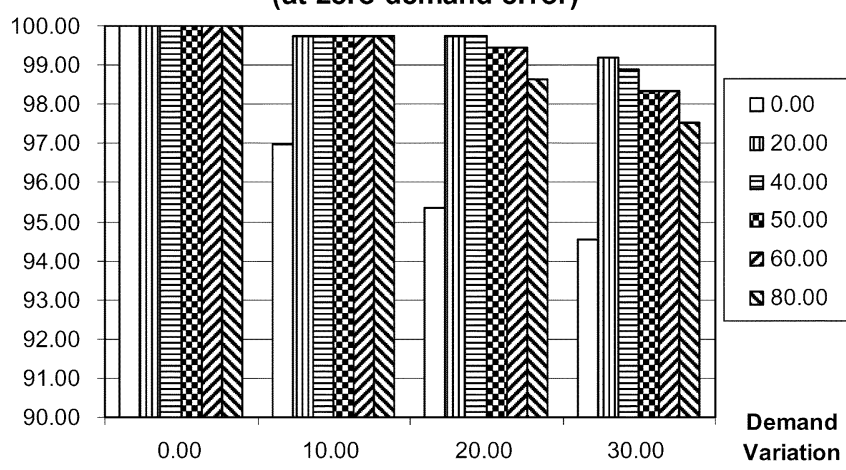

(Remark: the values of the legend are the EOCQ value in percentage of the EOQ quantity)

Fig. 4. Impact of demand variation on fill rate of the system with different EOCQ values (at zero demand error).

\section{Simulation Results With Coordination-With Multiple EOCQ Values}

Similar study as stated in Section IV-B has been conducted with two EOCQ values. The results are summarized in Table VI.
TABLE VI

PERCENTAGE IMPROVEMENT IN TOTAL UNIT COST WITH TwO EOCQ vaLUES (a) $E O C Q_{1}$ values $=30 \%$ of $\mathrm{EOQ} \mathrm{AND} E O C Q_{2}$ value $=50 \%$ of $\mathrm{EOQ}$; (b) $E O C Q_{1}$ value $=30 \%$ of EOQ AND $E O C Q_{2}$ value $=70 \%$ of $E O Q$; (c) $E O C Q_{1}$ value $=30 \%$ of $\mathrm{EOQ}$ AND $E O C Q_{2}$ value $=90 \%$ of $\mathrm{EOQ}$; (d) $E O C Q_{1}$ value $=50 \%$ of $\mathrm{EOQ}$ AND $E O C Q_{2}$ value $=70 \%$ of $\mathrm{EOQ}$; (e) $E O C Q_{1}$ value $=50 \%$ of $\mathrm{EOQ} \mathrm{AND} E O C Q_{2}$ value $=90 \%$ of $\mathrm{EOQ}$

\begin{tabular}{|c|c|c|c|c|}
\cline { 2 - 5 } \multicolumn{1}{c|}{} & \multicolumn{4}{c|}{ Demand Variation } \\
\hline Demand Error & $0 \%$ & $10 \%$ & $20 \%$ & $30 \%$ \\
\hline $0 \%$ & 0.00 & 1.24 & 1.25 & 1.11 \\
\hline $10 \%$ & 0.37 & 0.38 & 0.40 & 0.43 \\
\hline $20 \%$ & $\mathbf{- 0 . 1 0}$ & $\mathbf{- 0 . 1 0}$ & $\mathbf{- 0 . 1 0}$ & $\mathbf{- 0 . 1 0}$ \\
\hline $30 \%$ & $\mathbf{- 0 . 4 4}$ & $\mathbf{- 0 . 4 4}$ & $\mathbf{- 0 . 4 3}$ & $\mathbf{- 0 . 4 3}$ \\
\hline
\end{tabular}

(a)

\begin{tabular}{|c|c|c|c|c|}
\cline { 2 - 5 } \multicolumn{1}{c|}{} & \multicolumn{4}{c|}{ Demand Variation } \\
\hline Demand Error & $0 \%$ & $10 \%$ & $20 \%$ & $30 \%$ \\
\hline $0 \%$ & 0.00 & 1.24 & 1.25 & 1.11 \\
\hline $10 \%$ & 0.34 & 0.36 & 0.38 & 0.41 \\
\hline $20 \%$ & $\mathbf{- 0 . 1 3}$ & $\mathbf{- 0 . 1 3}$ & $\mathbf{- 0 . 1 2}$ & $\mathbf{- 0 . 1 2}$ \\
\hline $30 \%$ & $\mathbf{- 0 . 4 6}$ & $\mathbf{- 0 . 4 6}$ & $\mathbf{- 0 . 4 5}$ & $\mathbf{- 0 . 4 5}$ \\
\hline
\end{tabular}

(b)

\begin{tabular}{|c|c|c|c|c|}
\cline { 2 - 5 } \multicolumn{1}{c|}{} & \multicolumn{4}{c|}{ Demand Variation } \\
\hline Demand Error & $0 \%$ & $10 \%$ & $20 \%$ & $30 \%$ \\
\hline $0 \%$ & 0.00 & 1.24 & 1.23 & 1.11 \\
\hline $10 \%$ & 0.39 & 0.40 & 0.42 & 0.45 \\
\hline $20 \%$ & $\mathbf{- 0 . 0 8}$ & $\mathbf{- 0 . 0 8}$ & $\mathbf{- 0 . 0 8}$ & $\mathbf{- 0 . 0 8}$ \\
\hline $30 \%$ & $\mathbf{- 0 . 4 2}$ & $\mathbf{- 0 . 4 2}$ & $\mathbf{- 0 . 4 2}$ & $\mathbf{- 0 . 4 1}$ \\
\hline
\end{tabular}

(c)

\begin{tabular}{|c|c|c|c|c|}
\cline { 2 - 5 } \multicolumn{1}{c|}{} & \multicolumn{4}{c|}{ Demand Variation } \\
\hline Demand Error & $0 \%$ & $10 \%$ & $20 \%$ & $30 \%$ \\
\hline $0 \%$ & 0.00 & 1.12 & 0.80 & 0.51 \\
\hline $10 \%$ & $-\mathbf{- 1 . 7 7}$ & $-\mathbf{1 . 7 7}$ & $\mathbf{- 1 . 7 5}$ & $\mathbf{- 1 . 7 2}$ \\
\hline $20 \%$ & $\mathbf{- 1 . 7 0}$ & $\mathbf{- 1 . 7 0}$ & $\mathbf{- 1 . 7 0}$ & $\mathbf{- 1 . 7 0}$ \\
\hline $30 \%$ & $\mathbf{- 1 . 6 5}$ & $\mathbf{- 1 . 6 5}$ & $\mathbf{- 1 . 6 5}$ & $\mathbf{- 1 . 6 5}$ \\
\hline
\end{tabular}

(d)

\begin{tabular}{|c|c|c|c|c|}
\cline { 2 - 5 } \multicolumn{1}{c|}{} & \multicolumn{4}{c|}{ Demand Variation } \\
\hline Demand Error & $0 \%$ & $10 \%$ & $20 \%$ & $30 \%$ \\
\hline $0 \%$ & 0.00 & 1.12 & 0.80 & 0.51 \\
\hline $10 \%$ & $\mathbf{- 1 . 7 5}$ & $\mathbf{- 1 . 7 5}$ & $\mathbf{- 1 . 7 4}$ & $\mathbf{- 1 . 7 1}$ \\
\hline $20 \%$ & $\mathbf{- 1 . 6 8}$ & $\mathbf{- 1 . 6 8}$ & $\mathbf{- 1 . 6 8}$ & $-\mathbf{- 1 . 6 8}$ \\
\hline $30 \%$ & $\mathbf{- 1 . 6 3}$ & $\mathbf{- 1 . 6 3}$ & $\mathbf{- 1 . 6 3}$ & $\mathbf{- 1 . 6 4}$ \\
\hline
\end{tabular}

(e)

Again, to ease the analysis, the two EOCQ values (should be $E O C Q_{1}$ and $E O C Q_{2}$ with respect to (3)) are expressed in percentage of the EOQ value as discussed in Section IV-B. Only total cost is listed because similar findings as in Section IV-B could be found in the cases with multiple EOCQ values. Therefore, they are omitted.

Refer to Table VI, improvement could also be found when two EOCQ values are considered. However, improvement is more significant if all the two parameters are set higher than half of the EOQ values. This can be illustrated easily if Table VI(a) to $\mathrm{VI}(\mathrm{c})$ are grouped as GROUP 1 while Table VI(d) to VI(e) are grouped as GROUP 2. Main characteristic (and hence difference) of the two groups is that $E O C Q_{1}$ of each table in GROUP 1 is lower than half of the EOQ value; while in GROUP 2, all $E C O Q_{1}$ and $E O C Q_{2}$ values are higher than or equal to half of the EOQ value. From GROUP 1 (i.e., Table VI(a) to Table VI(c)), it could be concluded that if the contract can be completed more easily because of the small $E O C Q_{1}$ value, improvement could only be found in high demand errors settings regardless of the demand variation. In fact, the same argument as discussed in Section IV-B1 applies-this is related to a 
dilemma that when the rule is triggered, back order cost can be reduced while additional management costs are incurred. Therefore, if the $E O C Q_{1}$ value is too low, the additional management cost cannot be compensated by the reduction in back order cost. Therefore, low EOCQ values are not desired, which could be proven in GROUP 2 [i.e., Table VI(d) and (e)].

In fact, more sensitivity analysis has been conducted with multiple EOCQ values (like three and four EOCQ values). However, similar conclusion as discussed above could be drawn so the numerical values are omitted.

\section{CONClusions ANd Suggestions of Future Research}

\section{A. Discussions and Conclusions}

A coordination mechanism with early order completion contract is proposed in this paper to react with demand uncertainty in a distributed supply chain. Simulation results show that different performance measures can be improved against different EOCQ value of the proposed algorithm. However, as a rule of thumb, the EOCQ value can be set approximately as half of the EOQ quantity, i.e., $40 \%$ to $60 \%$, in order to gain the maximum benefit when demand error is non zero. When demand error is zero, a dilemma has been encountered to improve the fill rate by adopting the algorithm or do nothing to maintain a relatively low costs impact due to demand variation. This rule of thumb, however, is concluded according to the simulation results that are presented in this paper. In order to strength this conclusion, it is worth to perform sensitivity analysis in the future to simulate different settings (e.g. cost structure).

In fact, the simulation result in this paper is just a piece of evidence to support the proposed algorithm. The most important implication that can be derived from this paper, with support by the simulation results, is that introduction of flexibility (in the form of contract in this paper) is able to reduce the impacts of uncertainties. With flexibility in order quantity as established in the contract, demand uncertainty can be "digested" through the interactive coordination procedures. This is because the flexibility in the contract overcomes the variations of demand (or in general, local situations). As a matter of fact, such uncertainties are not easy to be represented by mathematically modelling which means traditional optimization techniques are not applicable to improve the performance of such systems effectively. With distributed configuration as in this simulation study, the situation can be more complex because independent agents (i.e., companies) may not be cooperative because of self-interest. Through the contract approach, behavior of agents is confined by contract which does not require complete information sharing. Therefore, a compromised solution can be reached interactively through the coordination mechanism.

\section{B. Limitations and Suggestions of Future Research}

One of the limitations of this research is that participating companies in the supply chain under study are willing to be coordinated according to the proposed algorithm. In fact, the algorithm only enforces some managerial actions and hence this assumption is easy to achieve. Another limitation is that only EOQ policy is compared with the proposed coordinated approach.
On one hand probabilities-based policy can be studied as mentioned. On the other hand, the proposed coordination algorithm does nothing in system optimization. It serves as a fine adjustment from the optimized setting, which can be obtained from any optimization techniques, in order to reduce the degree of deterioration of the system under uncertainty, which is usually not considered in traditional optimization approaches as found in the literature. In this connection, the concept can be adopted by any optimization techniques.

From the implications of the research as discussed above, future research can take the following factors into consideration.

- More sophisticated demand patterns (e.g., like Markov Chains) and more sensitivity analysis could be performed in order to verify the usefulness of the concept;

- Include more uncertain parameters-Since this study pinpoints the impacts of positive demand error, other uncertainties like error and variation in suppliers' capacity level can be added on top of this study for evaluation. In addition, performance of the contract approach under combinations of demand and capacity variation is also an interesting environment to be studied.

- Compare the coordination mechanism with stochastic (i.e., probabilistic-based) inventory policy, instead of EOQ policy. In principle, stochastic inventory policy makes use of probability function to add safety stock in the system as a buffer to counter react with uncertainties. Therefore, stochastic inventory policy should work better than EOQ policy subject to uncertainties as set in this study. It is valuable to evaluate the performance of the proposed contract approach with the stochastic inventory policy.

Since the contract can be defined as a constraint, or a set of constraints, the philosophy is in line with solving Constraint Satisfaction Problem (CSP). An application of CSP by taking above factors into considerations could be found in some preliminary studies by the authors [27], [28], who formulate and solve above problems through a CSP-based coordination mechanism. Another possible extension of this research is to incorporate fuzzy logic into consideration (e.g. [8]). Fuzzy logic is a well-known tool to taking uncertainty into consideration by modelling uncertain parameters by linguistic values like "less," "more," "most," and "full,", while the EOCQ values in the proposed contract approach in this study is just like the linguistic values. However, decision-making is based on fuzzy membership functions, which should be determined beforehand. In distributed environment, it is not possible to send complicated fuzzy membership functions among members since there are too many varieties of the functions. Chan and Chan [29] took an initiative to discuss a multi-agent based framework for sending fuzzy membership functions to other members in a supply chain for making decision. Although their proposed framework can reduce the information to be transmitted among agents by using a generic representation of the fuzzy membership functions, associated decision-making algorithms have not been discussed in determining the EOCQ values in their study. This is certainly a research direction for further study in finding such values based on fuzzy logic tools. 


\section{REFERENCES}

[1] M. Christopher, Logistics and Supply Chain Management. London, U.K.: Pitman, 1998.

[2] A. Villa, "Temporary network of enterprises: managing complexity by a multi-agent architecture," Proc. Inst. Mech. Eng. B: J. Eng. Manufact., vol. 216, pp. 611-616, 2002.

[3] M. Fisher, J. Hammond, W. Obermeyer, and A. Raman, "Configuring a supply chain to reduce the cost of demand uncertainty," Prod. Oper. Manag., vol. 6, pp. 211-225, 1997.

[4] M. Milgate, "Supply chain complexity and delivery performance: an international exploratory study," Supply Chain Manag.: Int. J., vol. 6, pp. 106-118, 2001.

[5] S. H. Huan, S. K. Sheoran, and G. Wang, "A review and analysis of supply chain operations reference (SCOR) model," Supply Chain Manag.: Int. J., vol. 9, pp. 23-29, 2004.

[6] D. Petrovic, "Simulation of supply chain behaviour and performance in an uncertain environment," Int. J. Prod. Econ., vol. 71, pp. 429-438, 2001.

[7] P. Kouvelis and J. M. Milner, "Supply chain capacity and outsourcing decisions: The dynamic interplay of demand and supply uncertainty," IIE Trans., vol. 34, pp. 717-728, 2002.

[8] D. Petrovic, R. Roy, and R. Petrovic, "Supply chain modelling using fuzzy sets," Int. J. Prod. Econ., vol. 59, pp. 443-453, 1999.

[9] L. Geneste, B. Grabot, and A. Letouzey, "Scheduling uncertain orders in the customer-subcontractor context," Eur. J. Oper. Res., vol. 147, pp. 297-311, 2003.

[10] M. Pagell and C. Sheu, "Buyer behaviours and the performance of the supply chain: an international exploration," Int. J. Prod. Res., vol. 39, pp. 2783-2801, 2001.

[11] I. Giannoccaro, P. Pontrandolfo, and B. Scozzi, "A fuzzy echelon approach for inventory management in supply chains," Eur. J. Oper. Res., vol. 149, pp. 185-196, 2003.

[12] X. Zhao and J. Xie, "Forecasting errors and the value of information sharing in a supply chain," Int. J. Prod. Res., vol. 40, pp. 311-335, 2002.

[13] X. Zhao, J. Xie, and R. S. M. Lau, "Improving the supply chain performance: use of forecasting models versus early order commitments," Int. J. Prod. Res., vol. 39, pp. 3923-3939, 2001.

[14] X. Zhao, J. Xie, and J. Leung, "The impact of forecasting model selection on the value of information sharing in a supply chain," Eur. $J$. Oper. Res., vol. 142, pp. 321-344, 2002.

[15] M. Rudberg and J. Olhager, "Manufacturing networks and supply chains: an operations strategy perspective," OMEGA: Int. J. Manag. Sci., vol. 31, pp. 29-39, 2003.

[16] H. L. Lee, V. Padmanabhan, and S. Whang, "Information distortion in a supply chain: the bullwhip effect," Manag. Sci., vol. 43, pp. 546-558, 1997.

[17] Z. K. Weng and T. McClurg, "Coordinated ordering decisions for short life cycle products with uncertainty in delivery time and demand," Eur. J. Oper. Res., vol. 151, pp. 12-24, 2003.

[18] J. Chen and L. Xu, "Coordination of the supply chain of seasonal products," IEEE Trans. Syst., Man, Cybern. A, Syst. Humans, vol. 31, no. 6, pp. 524-531, Nov. 2001.

[19] A. A. Tsay, "The quantity flexibility contract and supplier-customer incentives," Manag. Sci., vol. 45, pp. 1339-1358, 1999.

[20] F. T. S. Chan and H. K. Chan, "The future trend on system-wide modelling in supply chain studies," Int. Jo. Adv. Manufact. Technol., vol. 25, pp. 820-832, 2005.

[21] D. J. Wu, "Software agents for knowledge management: coordination in multi-agent supply chains and auctions," Exp. Syst. Applicat., vol. 20, pp. 51-64, 2001.

[22] J.-J. Jung and G.-S. Jo, "Brokerage between buyer and seller agents using constraint satisfaction problem models," Decision Support Syst., vol. 28, pp. 293-304, 2000.

[23] S. O. Kimbrough, D. J. Wu, and F. Zhong, "Computers play the beer game: can artificial agents manage supply chains?," Decision Support Syst., vol. 33, pp. 323-333, 2002.
[24] J. Sterman, "Modeling managerial behavior: misperceptions of feedback in a dynamic decision making experiment," Manag. Sci., vol. 35, pp. 321-339, 1989.

[25] F. T. S. Chan and H. K. Chan, "A new model for manufacturing supply chain networks: a multiagent approach," Proc. Inst. Mech. Eng. B: J. Eng. Manufact., vol. 218, pp. 443-454, 2004.

[26] J. R. Evans, Production/Operations Management: Quality, Performance, and Value. Minneapolis, MN: West, 1997.

[27] H. K. Chan and F. T. S. Chan, "A coordination framework for distributed supply chains," in Proceedings of the IEEE Int. Conf. Systems, Man and Cybernetics, The Hague, The Netherlands, Oct. 10-13, 2004, pp. 4535-4540.

[28] H. K. Chan, F. T. S. Chan, and P. Samaranayake, "Adaptive make-to-order strategy in distributed manufacturing supply chains," in CD Proc. 3rd Int. IEEE Conf. Industrial Informatics, Perth, Australia, Aug. 10-12, 2005.

[29] F. T. S. Chan and H. K. Chan, "Fuzzy multi-agent based supply chain networks models," in CD Proc. 9th Int. Conf. Manufacturing Excellence, Melbourne, Australia, Oct. 13-15, 2003.

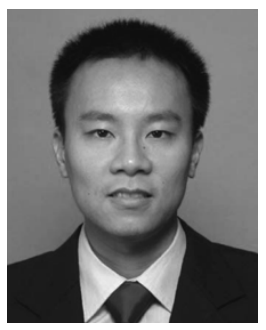

Hing Kai Chan (M'98-SM'04) received the B.Eng. degree in electrical and electronic engineering and the M.Sc. degree in industrial engineering and industrial management, both from the University of Hong Kong, in 1995 and 2000, respectively. He is currently pursuing the Ph.D. degree at the University of Hong Kong.

Previously, he was a Design and Project Engineer, with focus on instrumentation and measurement, in the electronic manufacturing sector. His current research interests include industrial informatics and applications of soft computing on intelligent industrial systems and supply chains. Mr. Chan is a recipient of the IEEE Industrial Electronics Society Student Travel Grant in 2005. He is an Organizing Committee Member of the IEEE Region Ten Annual Technical Conference (TENCON) 2006, and is an International Technical Programme Committee Member of the IEEE International Conference on Industrial Informatics 2006. He is a member of the Institution of the Electrical Engineers, and the Chartered Institute of Marketing, U.K. He is a Chartered Engineer and a Chartered Marketer, UK.

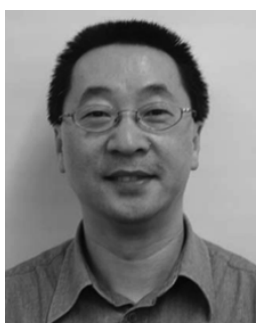

Felix T. S. Chan received the B.Sc. degree (with First Class Honour) in mechanical engineering from Brighton Polytechnic (now University), U.K., and the $\mathrm{Ph} . \mathrm{D}$. degree in manufacturing engineering from the Imperial College of Science and Technology, University of London, U.K.

He was a Research Fellow for two years in the Department of Design, Manufacture and Engineering Management, University of Strathclyde, U.K. Prior to joining The University of Hong Kong in 1996, he was a Senior Lecturer at the School of Manufacturing and Mechanical Engineering, University of South Australia. He is now an Associate Professor, Department of Industrial and Manufacturing Systems Engineering, The University of Hong Kong. His current research interests are logistics and supply chain management, distribution coordination, systems modelling and simulation, and supplier selection. To date, he has published three book chapters, over 150 refereed international journal papers and 160 peer reviewed international conference papers.

Dr. Chan is a senior member of the Society of Manufacturing Engineers, and a chartered member of the Chartered Institute of Logistics and Transport in Hong Kong. 